\title{
HISTOPLASMA STOMATITIS UNVEILED: Not all opportunistic infections get better after initiation of antiretroviral therapy
}

\author{
Saurabh Bansal ${ }^{1}$, Namrata Singhania ${ }^{2}$, Chandra Nukala ${ }^{3}$, Anil Singh ${ }^{4}$, and Laith Al \\ Rabadi $^{5}$ \\ ${ }^{1}$ University of Illinois College of Medicine at Peoria \\ ${ }^{2}$ Mount Carmel East \\ ${ }^{3} \mathrm{CHI}$ St Vincent Infirmary \\ ${ }^{4}$ Geisinger-Community Medical \\ ${ }^{5}$ University of Utah Health
}

November 2, 2020

\begin{abstract}
Histoplasmosis occurs primarily in the lungs but can disseminate in immunocompromised patients. Serology can be negative in patients with local infection, making the diagnosis challenging. Definite diagnosis is by microscopic examination of the tissue sample. We report a rare case of Histoplasma stomatitis whose lesions manifested after initiating antiretroviral therapy.

Type of article: Case Report

Title of the article: HISTOPLASMA STOMATITIS UNVEILED: Not all opportunistic infections get better after initiation of antiretroviral therapy

Running title: Histoplasma stomatitis in HIV patient

Contributors

1. Bansal, Saurabh MD

2. Singhania, Namrata $\mathrm{MD}^{\mathrm{b}}$

3. Nukala, Chandra Mouli $\mathrm{MD}^{\mathrm{c}}$

4. Singh, Anil Kumar MD ${ }^{d}$

5. Al-Rabadi, Laith $\mathrm{MD}^{\mathrm{e}}$

Department(s) and institution(s)

${ }^{a}$ Department of Internal Medicine, University of Illinois College of Medicine at Peoria, Peoria IL USA 61637

${ }^{\mathrm{b}}$ Department of Hospital Medicine, Mount Carmel East Hospital, Columbus OH USA 43213

${ }^{\mathrm{c}}$ Department of Hospital Medicine, CHI St Vincent Infirmary, Little Rock AR USA 72205

${ }^{\mathrm{d}}$ Department of Internal Medicine, Geisinger Community Medical Center, Scranton, PA USA 18510

e Division of Nephrology, University of Utah, Salt Lake City, Utah USA 84132

Corresponding Author:
\end{abstract}


Full name: Laith Al-Rabadi

Department/University: University of Utah

Street Name \& Number: 30 N Medical Drive, Rm 4R312

City, State, Country, Postal code: Salt Lake City, Utah, USA, 84132

Tel: +1-319-855-8439; Fax: +1-801-581-4343; E-mail: laithalrabadi3@gmail.com

Total number of photographs: 1

Word counts

for abstract: 50 words

for the text: 996 words

Funding source(s) of support: None to report

Conflict of Interest: There are no conflict of interest to disclose

Title of the article: HISTOPLASMA STOMATITIS UNVEILED: Not all opportunistic infections get better after initiation of antiretroviral therapy

Abstract:

Histoplasmosis occurs primarily in the lungs but can disseminate in immunocompromised patients. Serology can be negative in patients with local infection, making the diagnosis challenging. Definite diagnosis is by microscopic examination of the tissue sample. We report a rare case of Histoplasma stomatitis whose lesions manifested after initiating antiretroviral therapy.

Keywords: Histoplasma, HIV, stomatitis, IRIS, antiretroviral therapy

Key Message:

Immune reconstitution syndrome in AIDS patients can lead to initial worsening of underlying diseases due to body's ability to mount a strong immune response after recovery of CD4 counts.

Introduction:

Histoplasma Capsulatum is a dimorphic, round, budding yeast that is found primarily along the Mississippi and Ohio River valleys in the United States but can be seen in other areas also. ${ }^{1}$ Infection occurs by inhalation of conidia, which primarily manifests initially as pulmonary infection, but via hematogenous route, dissemination can occur, especially in high-risk immunocompromised patients. Dissemination occurs in extra-pulmonary organs causing gastrointestinal infection, stomatitis, mucositis, central nervous system, or bone marrow infection. ${ }^{2}$ Immune reconstitution inflammatory syndrome (IRIS) is a phenomenon in which there is paradoxical worsening of pre-existing disease or unmasking of subclinical disease after starting antiretroviral therapy (ART) due to improvement in immune response of the body. ${ }^{3,4}$ Muco-cutaneous lesions due to $\mathrm{H}$. capsulatum have been rarely associated with IRIS. ${ }^{5-7}$ We herein report a case of $\mathrm{H}$. capsulatum stomatitis in a HIV infected patient after resumption of his ART.

Case History:

A 34-year-old African American male with a past medical history of hemophilia A requiring twice-weekly recombinant Factor-VIII infusions, HIV-1, and Hepatitis C infection presented with 3-month history of intermittent fever, significant weight loss, and worsening lip swelling and ulceration. He became compliant with ART 6 months ago as evident by improvement in CD4 count from 94 cells $/ \mathrm{mm}^{3}$ to $160 \mathrm{cells} / \mathrm{mm}^{3}$. His muco-cutaneous lesions appeared two months after improvement in CD4 count. He received multiple antibiotics and steroids in the past with no relief. He was on acyclovir and dapsone for Herpes Simplex virus (HSV) and Pneumocystis jiroveci prophylaxis, respectively. Physical examination revealed significantly 
swollen lips and multiple superficial ulcers with heaped-up margins (Figure 1). No obvious hepatomegaly or splenomegaly was noted. Differential diagnosis included herpetic ulcers, bacterial infections, fungal infections or angioedema. He was initially started on valacyclovir for presumed HSV infection. HSV polymerase chain reaction taken from the base of lip ulcer was negative. HIV \& Hepatitis-C viral loads were undetectable. Complement C1-esterase inhibitor level was negative. Gram stain, acid-fast stain, mucicarmine stain, fungal blood cultures, urine and serum Histoplasma antigen, were negative. Complement fixation test was positive for H. capsulatum mycelial and yeast forms (titer $>1: 256$ ). Serum immunodiffusion assay was strongly positive for $\mathrm{H}$. capsulatum ' $\mathrm{H}$ ' and ' $\mathrm{M}$ ' bands but negative for Aspergillus fumigatus, Blastomyces dermatiditis and Coccidiodes immitis. Biopsy of the lips revealed granulomatous and mixed inflammatory infiltrate predominantly with lymphocytes with areas of necrosis and presence of small budding yeast forms. Patient was diagnosed with $\mathrm{H}$. capsulatum associated stomatitis and was started on itraconazole for total twelve months. He demonstrated dramatic regression of oral edema and ulcerations at six-month follow up visit.

Discussion:

Histoplasmosis is the most common endemic mycosis in AIDS patients, frequently observed in Mississippi and Ohio River valley. ${ }^{1}$ Incidence is reportedly 3.4 cases per 100,000 person-years in the United States. It usually manifests as primary pulmonary infection from inhalation of microconidia but can disseminate hematogenously in immunocompromised patients - leading to skin, neurological, bone marrow or gastrointestinal abnormalities. ${ }^{2}$ The most common symptoms of pulmonary histoplasmosis are fever, chills, weakness and cough with chest radiograph commonly revealing interstitial or reticulo-nodular infiltrates. Other findings seen on chest imaging are pneumonia-like consolidation with or without hilar lymphadenopathy, and/or cavitary lung lesion. Disseminated histoplasmosis patients often have progressive weight loss and fever with other clinical symptoms depending on the organ system involved. ${ }^{8}$

Diagnosing histoplasmosis in mild to moderate local infection can be challenging. Serum and urine antigen testing and tissue cultures may remain negative. Definite diagnosis is achieved by microscopic examination of tissue sample which can reveal immature forms. ${ }^{9}$ Both the immunodiffusion test and the complement fixation test should be used for workup. Antibody detection of ' $\mathrm{H}$ ' \& ' $\mathrm{M}$ ' bands through immunodiffusion has higher sensitivity than complement fixation. Titers $>1: 32$ or increasing titers when checked at an interval of 1-2 weeks is highly suggestive of $\mathrm{H}$. capsulatum infection. ${ }^{9,10}$ Antibody testing can have low sensitivity in immunocompromised patients. ${ }^{10}$ New emerging tests such as microbial cell-free DNA testing can have quick turnaround time and help in diagnosing the disease sooner. ${ }^{11}$

Unveiling of manifestations of histoplasmosis as a result of Immune reconstitution inflammatory syndrome (IRIS) has been rarely reported in literature. ${ }^{5-7}$ Patients with active HIV infection who are not taking ART, have low CD4 count, high viral load and poor immune response. ${ }^{12}$ Rapid improvement in immune function and suppression of viral load after initiation of ART can lead to systemic or local inflammatory response at sites of preexisting infection which were not clinically apparent before initiation of ART. Increases in $\mathrm{T}$ lymphocytes after initiation of ART activates suppressed immune response leading to inflammation and granuloma formation. This can happen in 1 to 3 months after initiation of ART. ${ }^{13}$ This phenomenon is more commonly reported in patients with tuberculosis; therefore, tuberculosis remains main differential in suspected IRIS patients.

Our patient developed his lesions two months after he started taking ART as evident from his improvement in CD4 counts and suppression of HIV viral load. Strong immune response to localized H. capsulatum infection as evidenced by both immunodiffusion and complement fixation assays is also compelling. Treatment of histoplasmosis depends on clinical form and severity and involves various antifungals like azole drugs in mild to moderate disease or amphotericin B in moderate to severe cases. ${ }^{14}$ Also, immunocompromised patients with severe disease at presentation are frequently treated with amphotericin B. In patients with severe disease, after initial induction phase of one to two weeks with amphotericin B, treatment can be switched to oral azole drugs like itraconazole. The preferred duration for mild to moderate infection is 12 months and longer for severe disease, keeping in mind, lifelong therapy may be needed in selected individuals. ${ }^{15}$ Monitoring of drug levels is recommended to ascertain compliance and adequate levels. Histoplasma antigen 
levels are frequently used to monitor treatment response. For treatment of IRIS, use of glucocorticoids, which may seem counter-intuitive in an immunocompromised patient with disseminated infection, may be needed in selected individuals.

In summary, H. capsulatum associated stomatitis can be diagnosed by microscopic examination of tissue sample along with immunodiffusion and complement fixation tests. Antigen test can be negative in localized infection. It can be associated with IRIS with paradoxical worsening of mucocutaneous lesions after initiation of ART.

Acknowledgement: None

Author contributions: SB and NS contributed equally in preparing the manuscript and reviewed the literature. CMN, AKS and LA critically revised the manuscript.

Ethical Approval: Ethic committee was not consulted for approval as it is a case report and all possible efforts were made to maintain complete anonymity.

References:

1. Benedict K, Mody RK. Epidemiology of Histoplasmosis Outbreaks, United States, 1938-2013. Emerg Infect Dis . 2016;22(3):370-378. doi:10.3201/eid2203.151117

2. Goodwin RA, Shapiro JL, Thurman GH, Thurman SS, Prez RMD. Disseminated histoplasmosis: Clinical and pathologic correlations. Med (United States) . 1980;59(1):1-33. doi:10.1097/00005792-198001000-00001

3. Mayer KH, Hirsch HH, Kaufmann G, Sendi P, Battegay M. Immune Reconstitution in HIV-Infected Patients. Clin Infect Dis . 2004;38(8):1159-1166. doi:10.1086/383034

4. French MA. HIV/AIDS: immune reconstitution inflammatory syndrome: a reappraisal. Clin Infect Dis . 2009;48(1):101-107. doi:10.1086/595006

5. Melzani A, de Reynal de Saint Michel R, Ntab B, et al. Incidence and Trends in Immune Reconstitution Inflammatory Syndrome Associated With Histoplasma capsulatum Among People Living With Human Immunodeficiency Virus: A 20-Year Case Series and Literature Review. Clin Infect Dis . 2020;70(4):643-652. doi:10.1093/cid/ciz247

6. Kiggundu R, Nabeta HW, Okia R, Rhein J, Lukande R. Unmasking histoplasmosis immune reconstitution inflammatory syndrome in a patient recently started on antiretroviral therapy. Autops Case Reports . 2016;6(4):27-33. doi:10.4322/acr.2016.048

7. Dellière S, Guery R, Candon S, et al. Understanding pathogenesis and care challenges of immune reconstitution inflammatory syndrome in fungal infections. J Fungi . 2018;4(4). doi:10.3390/jof4040139

8. Wheat LJ. Improvements in diagnosis of histoplasmosis. Expert Opin Biol Ther . 2006;6(11):1207-1221. doi:10.1517/14712598.6.11.1207

9. Azar MM, Hage CA. Laboratory diagnostics for histoplasmosis. J Clin Microbiol . 2017;55(6):1612-1620. doi:10.1128/JCM.02430-16

10. Hage CA, Ribes JA, Wengenack NL, et al. A multicenter evaluation of tests for diagnosis of histoplasmosis. Clin Infect Dis . 2011;53(5):448-454. doi:10.1093/cid/cir435

11. Bansal S, Yadav M, Singhania N, Samal S, Singhania G. Blastomycosis Detected by Microbial Cell-Free DNA in Renal Transplant Recipient.Am J Med . 2020. doi:10.1016/j.amjmed.2020.02.043

12. Antonelli LR V, Mahnke Y, Hodge JN, et al. Elevated frequencies of highly activated CD4+ T cells in HIV+ patients developing immune reconstitution inflammatory syndrome. Blood . 2010;116(19):3818-3827. doi:10.1182/blood-2010-05-285080 
13. Murdoch DM, Venter WDF, Feldman C, Van Rie A. Incidence and risk factors for the immune reconstitution inflammatory syndrome in HIV patients in South Africa: a prospective study. AIDS . 2008;22(5):601-610. doi:10.1097/QAD.0b013e3282f4a607

14. Wheat LJ, Freifeld AG, Kleiman MB, et al. Clinical Practice Guidelines for the Management of Patients with Histoplasmosis: 2007 Update by the Infectious Diseases Society of America. Clin Infect Dis . 2007;45(7):807-825. doi:10.1086/521259

15. Wheat LJ, Cloud G, Johnson PC, et al. Clearance of fungal burden during treatment of disseminated histoplasmosis with liposomal amphotericin B versus itraconazole. Antimicrob Agents Chemother . 2001;45(8):2354-2357. doi:10.1128/AAC.45.8.2354-2357.2001

Figure legend:

Figure 1. Histoplasma associated stomatitis showing swollen lips and superficial ulcers with heaped up margins.

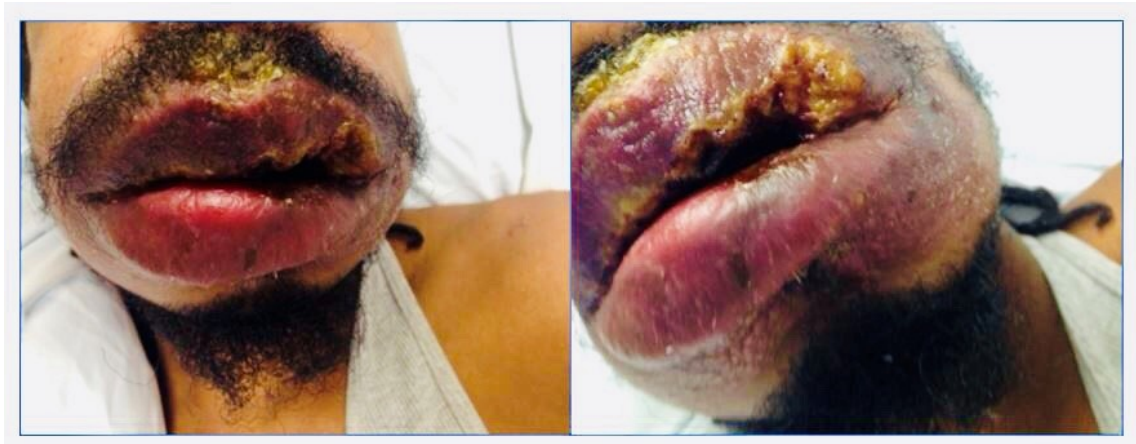

